Mappemonde

Revue trimestrielle sur l'image géographique et les formes du territoire

$121 \mid 2017$

Varia

\title{
Bande dessinée et cartographie : une imbrication créative
}

Comics and cartography: a creative imbrication

Cómic y cartografía : una interconexión creativa

\section{Vincent Veschambre}

\section{(2) OpenEdition}

Journals

Édition électronique

URL : http://journals.openedition.org/mappemonde/3511

DOI : 10.4000/mappemonde.3511

ISSN : 1769-7298

Éditeur

UMR ESPACE

Référence électronique

Vincent Veschambre, "Bande dessinée et cartographie : une imbrication créative », Mappemonde [En

ligne], 121 | 2017, mis en ligne le 01 juillet 2017, consulté le 29 avril 2020. URL : http://

journals.openedition.org/mappemonde/3511; DOI : https://doi.org/10.4000/mappemonde.3511

Ce document a été généré automatiquement le 29 avril 2020

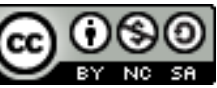

La revue Mappemonde est mise à disposition selon les termes de la Licence Creative Commons Attribution - Pas d'Utilisation Commerciale - Partage dans les Mêmes Conditions 4.0 International. 


\title{
Bande dessinée et cartographie : une imbrication créative
}

\author{
Comics and cartography: a creative imbrication \\ Cómic y cartografía : una interconexión creativa
}

Vincent Veschambre

« Tout récit suppose un espace, toute histoire nécessite un territoire. Cette géographie souvent

fictive des auteurs de bande dessinée est une création géopolitique »

(Douvry Jean-François, 1992. Grand Atlas des pays

imaginaires de la bande dessinée. Grenoble :

Phoenix).

\section{Introduction : art séquentiel, récit et spatialisation}

Dans son ouvrage sur les "portraits de ville", Henri Garric considère que "La représentation de la ville dans la civilisation occidentale se fait par l'association de trois types de la représentation, l'attribution (i.e le nom), la carte et le parcours (...). La carte organise les lieux les uns par rapport aux autres dans un rapport de coexistence et produit une première organisation du récit; mais surtout, le parcours met en place un véritable "récit de ville", animant la fixité de la carte et relayant les uns aux autres des lieux qui justement n'étaient posés que dans leur juxtaposition» (Garric, 2007, p. 74). Dans une conférence qu'il a tenue à l'ENS de Lyon en 2013, consacrée au thème "Ville et $\mathrm{BD}$ » $^{1}$, il a eu l'occasion de préciser que le genre de la bande dessinée était, selon lui, "le lieu le plus avancé de synthèse de ces différentes formes" que représentent le parcours (le principe dynamique du récit) et la carte (la description de l'espace dans lequel il se déploie). Pour illustrer cela, Henri Garric a cité l'œuvre des Cités obscures, cosignée par François Schuiten et Benoît Peeters, dans laquelle, à partir de déplacements des personnages, peuvent s'agencer vues perspectives, vues fragmentaires, vues aériennes, plans, cartes... 
2 Si l'on en croit Thierry Groensteen, l'un des principaux théoriciens du genre, la bande dessinée peut se définir comme "mise en relation d'une pluralité d'images solidaires » (Groensteen, 1999, p. 21). Et comme il le précise, « mettre en relation les vignettes de la bande dessinée, c'est [...] nécessairement mettre en relation des espaces » (ibid., p. 26) : c'est bien cette mise en relation des espaces qui est constitutive du récit dans la bande dessinée. Un mode de narration séquentiel qui peut être cependant relayé ou suspendu par cet autre mode de représentation graphique qu'est la carte. Fortement ancrée dans les espaces urbains, à l'image des sociétés dont elle est issue, la bande dessinée donne à lire des parcours dans la ville, par l'enchaînement des cases racontant le déplacement des personnages et le déroulement de l'action, avec une diversité de points de vue, mais également des cartes (Marlet, 2007), ou des plans, à plus grande échelle, qui permettent de localiser et de contextualiser cette action.

Ces réflexions théoriques sur les rapports entre narration et spatialisation m'inclinent à penser que la bande dessinée est un genre particulièrement pertinent pour s'interroger sur le lien entre cartographie et récit.

4 Je fais donc l'hypothèse que la carte joue un rôle prédominant, dans la bande dessinée. Par rapport à d'autres modes de narration, comme les différentes formes de littérature, la bande dessinée apparaît a priori mieux à même d'intégrer le langage graphique de la carte. La case de bande dessinée et la carte sont d'ailleurs toutes deux généralement cernées par un cadre, qui délimite l'espace de la représentation : comme le rappelle Thierry Groensteen, «il n'existe pas d'icône qui ne campe pas à l'intérieur de frontières" (ibid., p. 52). Même si la case s'inscrit dans une séquence narrative, tandis que la carte peut être lue isolément et se suffrre à elle-même.

5 Afin de préciser le rôle que joue la carte dans la narration en bande dessinée, j'ai travaillé sur deux corpus différents. En procédant tout d'abord à un sondage manuel (analyse de tous les ixièmes ouvrages dans les bacs) dans un fonds de bandes dessinées occidentales $^{2}$ (Amérique du Nord et Europe de l'Ouest) pour adultes, récemment éditées ${ }^{3}$, afin d'évaluer la proportion de BD comprenant des cartes et d'avoir un premier aperçu du type de cartes représentées. Cette première analyse sur un corpus de 100 BD ne visait pas l'exhaustivité quant au repérage des cartes, mais plutôt à signaler leur présence ou leur absence et à identifier les grands types de cartes utilisées.

6 Puis j'ai analysé dans le détail les cartes présentes dans un corpus de 50 BD occidentales issues de ma propre bibliothèque et que j'avais remarquées au fil des lectures, ce qui m'a permis de faire une analyse beaucoup plus fine, en identifiant toutes les cartes et en précisant systématiquement leur position dans le récit.

\section{À quoi sert la carte dans la BD ? Déclencheur et support du récit}

7 La première hypothèse à tester concerne cette idée d'un lien privilégié entre la bande dessinée et la carte. On peut tout d'abord faire le constat d'une présence de la carte dans de grands classiques du genre, tels que Les Aventures de Tintin, pour permettre de suivre ses voyages, Les Aventures d'Astérix le Gaulois, avec cette fameuse carte liminaire situant le village " qui résiste encore et toujours à l'envahisseur », ou encore avec Hugo 
Pratt qui ouvre la première aventure de Corto Maltese, La Ballade de la mer salée, par la carte d'un atoll du Pacifique.

\section{La carte hors-champ : « la possibilité d'un récit »}

8 Thierry Groensteen afFIrme que «Dans un tableau figuratif, même inanimé, on peut souvent voir déjà comme la promesse ou la possibilité d'un récit. L'imagination humaine a le pouvoir de discerner n'importe quelle icône en scénario potentiel» (Groensteen, 2003, p. 94).

9 Plusieurs témoignages d'auteurs de bande dessinée confirment cette puissance évocatrice de la carte pour construire leur récit, nous entraînant du côté de ces cartes hors champ qui ont été au point de départ de certains ouvrages ou de certaines séries. Ainsi pour la genèse de la série Philémon de Fred : «Si l'on en croit l'auteur, l'idée de cet univers original, mettant en scène les lettres de l'Atlantique, lui serait venue en regardant une simple mappemonde » (Gaumer, 1996, p. 151).

Prendre la carte « au pied de la lettre » : le Philémon de Fred

Dans le Naufragé du A, au début de la série Philémon de Fred (1972), le héros disparaît au fond d'un puits et réapparaît sur une terre étrange où le merveilleux s'impose à tout instant. Il rencontre Barthélemy, dans un costume de Robinson, le puisatier disparu il y a 40 ans.

$\mathrm{P}:$ Mais, enfin, où sommes-nous ici?

$B$ : Sur le « A » mon p'tit, nous sommes sur le A..

$\mathrm{P}:$ Le A ?

B : Eh oui, le «A »... remarque, j'ai mis des années avant de comprendre [...] Mais maintenant j'en suis sûr car j'ai aperçu le « $\mathrm{T}$ » et je sais que plus loin il y a le « $\mathrm{L}$ »... $\mathrm{P}: \mathrm{Qu}$ 'est-ce qu'il raconte ? Il déménage... ; cette longue solitude sans doute...

B : Non mon p'tit, je ne suis pas fou. Je vais te montrer. Barthélemy dessine du bout d'un bâton l'Atlantique nord entre Amérique du Nord et Europe et écrit "Océan atlantique » (p. 17-18).

$\mathrm{B}$ : Voici une carte du monde... enfin, je ne sais pas dessiner mais c'est à peu près ça... Ici se trouve l'Amérique, là l'Europe... et entre ces deux continents, l'Océan atlantique... eh ! bien nous sommes ici, sur le « A ».

La série Philémon procède d'une lecture poétique de la carte, d'une invention d'un monde à partir de la carte, prise littéralement « au pied de la lettre ».

En préambule du court récit Assemblage de traits, Alex Baladi (2009) raconte : «Quand j'étais petit, des dessins accrochés aux murs me fascinaient... Je voyais dans ces dessins des scènes étranges... je me racontais des histoires avec des personnages un peu tordus qui y étaient représentés ». Ces dessins accrochés aux murs, c'étaient des cartes, dont il fait aujourd'hui ressurgir les histoires autrefois imaginées (figure 1). 
Figure 1. Encore un effort

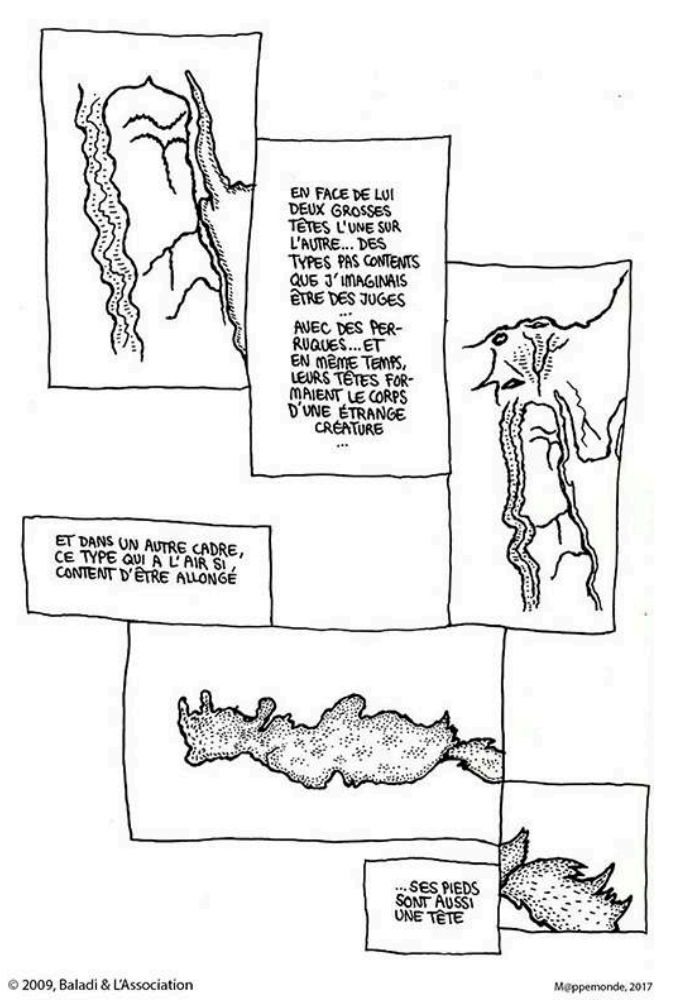

Non paginé.

2009, Baladi \& l'Association

11 Interviewé sur la place des cartes dans son travail (Les Maîtres cartographes, Troy), le scénariste Christophe Arleston déclare que ce n'est pas un hasard s'il y a une carte murale dans son atelier, héritée de ses grands-parents. Une carte du XVII ${ }^{\mathrm{e}}$ siècle de l'Asie qui l'a toujours fait rêver : " mon amour de fantasy vient aussi de là » (Bellefroid, 2008, p. 99). Dans la genèse du récit, la carte qu'il a réalisée joue un rôle essentiel : «Toute l'histoire de Troy est inscrite dans la carte qu'on a réalisée. Quand je la regarde, je trouve un scénario immédiatement. Parce qu'à travers la géographie, tu te dis : “tel fleuve passe par là donc tel peuple a des raisons d'y vivre et d'entrer en conflit avec tel autre. Tout découle automatiquement de la géographie” "(ibid., p. 99).

Dans le même ordre d'idée, Jean-Louis Tripp exprime l'importance de réaliser un plan pour construire un récit, celui de la série Magasin général, qui se déroule dans le Québec rural des années 1930. Avec Régis Loisel, ils n'avaient en tête que les deux scènes de départ: "Mais il faut bien l'avouer, une fois ces deux scènes écrites, nous ne savions plus trop dans quelle direction partir. C'est un peu pour cette raison que nous avons choisi de mieux définir le village dans lequel nous allions faire évoluer les personnages [...]. Régis a dessiné sur de grandes feuilles cet immense plan dont il vient de vous parler et moi, je me suis amusé à faire la généalogie de toutes les familles du village. Une fois ce travail fait, nous avions enfin en notre possession un village qui existait, dans lequel nous allions pouvoir faire évoluer nos personnages. Chaque matin, je m'installe à ma table et la porte du village s'ouvre... » ${ }^{4}$. Ce plan est d'ailleurs figuré sur les pages de garde de l'album: il a donc une fonction non seulement pour les auteurs, mais également pour les lecteurs qui sont censés entrer ainsi plus facilement dans cet univers. 


\section{Une carte bien présente dans la BD, avec des positions et des statuts différents}

13 Même si le potentiel narratif de la carte semble bien attesté, pour tout ce qui relève de la crédibilisation d'un récit, qu'il se situe dans un temps et un espace réalistes ou a fortiori dans un univers imaginaire, il m'a semblé essentiel d'y regarder de plus près et d'essayer d'évaluer plus précisément la présence de la carte dans la BD contemporaine. À travers l'échantillonnage auquel j'ai procédé dans le fonds de bandes dessinées étatsuniennes et européennes (principalement franco-belges), sur 100 BD consultées de manière aléatoire, j'ai constaté que plus de $40 \%$ des ouvrages consultés comportaient au moins une représentation cartographique, ce qui permet de parler d'une présence significative de la carte et me conforte dans l'idée d'analyser le rôle qu'elle joue dans la narration séquentielle.

Tableau 1. BD avec cartes du corpus 100 selon l'origine géographique du dessinateur

\begin{tabular}{|l|l|l|}
\hline Origine de l'auteur & Nombre avec cartes & Nombre total \\
\hline Franco-belge & 30 & 75 \\
\hline Européen & 2 & 10 \\
\hline États-unien & 10 & 13 \\
\hline Total & 42 & 100 \\
\hline
\end{tabular}

Source : échantillon 100 BD (Rize, 2014)

14 Même si les chiffres restent modestes et peu significatifs, il semblerait que la carte soit particulièrement bien représentée dans la BD états-unienne : cela mériterait en tout cas d'être vérifié à partir d'échantillons plus importants.

Pour tenter de répondre à cette question de la fonction de la carte dans la BD, j'ai tout d'abord cherché à la situer par rapport au récit séquentiel. Deux grands types de cartes peuvent être identifiés: les «cartes-représentation» et les «cartes-objet». Les premières ont pour fonction de représenter l'espace dans lequel s'inscrit le récit, ou de le situer, et correspondent à des icônes autonomes. Les deuxièmes correspondent à des objets, utilisés dans le récit et insérés dans les cases, au même titre que n'importe quel autre objet que croisent ou manipulent les personnages du récit.

\section{La carte-représentation : genèse et contextualisation du récit}

16 Le premier type de carte/plan permet une localisation, une contextualisation, donne une vision surplombante de l'espace du récit, à différentes échelles. Cela permet d'inscrire et de fonder le récit dans un espace, à travers sa représentation.

À l'intérieur de cette première catégorie, il faut distinguer la carte hors-récit de la carte insérée dans le récit. 


\section{La carte en amont ou en aval du récit}

Dans mes deux corpus, entre un tiers et la moitié des BD avec carte présente une carte hors-récit (tableau 2). Peu nombreuses quand elles sont présentes, mais généralement de grande taille, ces cartes ne représentent que $14 \%$ de l'échantillon total de cartes identifiées dans le corpus de 50 ouvrages.

Tableau 2. Poids des types de cartes dans les deux corpus (100 et 50 )

\begin{tabular}{|l|l|l|l|l|}
\hline & Avec cartes hors récit & Avec carte-case & Avec carte objet & Avec carte \\
\hline Nombre BD corpus 100 & 15 & 10 & 26 & 42 \\
\hline$\%$ BD avec carte (sur 42) & $36 \%$ & $24 \%$ & $62 \%$ & \\
\hline Nombre BD corpus 50 & 21 & 30 & 25 & 50 \\
\hline$\%$ & $42 \%$ & $60 \%$ & $50 \%$ & \\
\hline
\end{tabular}

Source : échantillon personnel de 50 BD avec cartes

19 Ce type de carte localise l'action, fournit le contexte du récit, le plus souvent a priori mais également a posteriori.

20 C'est la position occupée par la carte fameuse qui ouvre rituellement Les Aventures d'Astérix, et qui visualise l'argument du «village qui résiste encore et toujours... » La caricature et l'humour opèrent d'autant mieux que les auteurs se réfèrent à un contexte à la fois géographique et historique, qu'ils peuvent ensuite traiter de manière décalée. Dans le cas de François Bourgeon et des Passagers du vent (volume 3), le plan ancien du fort Saint-Louis de Juda (1776) est reproduit à la fois avant et après le récit: sa fonction est du même ordre, même s'il s'agit au contraire d'inscrire le récit dans une approche réaliste et de rendre les personnages crédibles. Rien de tel qu'une carte liminaire pour embrayer sur un récit totalement imaginaire, de type heroic fantasy, comme dans le cas de Lanfeust de Troy (volume 1), la série dessinée par Didier Tarquin et scénarisée par Christophe Arleston.

21 La carte peut également ouvrir le propos lorsqu'il s'agit inversement d'une approche documentaire, soit dans le genre historique (le Christophe Colomb dessiné par Enzo Biagi et Milo Manara) ou dans le genre reportage/récit de voyage, avec par exemple la carte du Cambodge (mais aussi la présentation des personnages, comme dans les Aventures d'Astérix) qui ouvre Palace, récit de voyage de Simon Hureau (figure 2). 


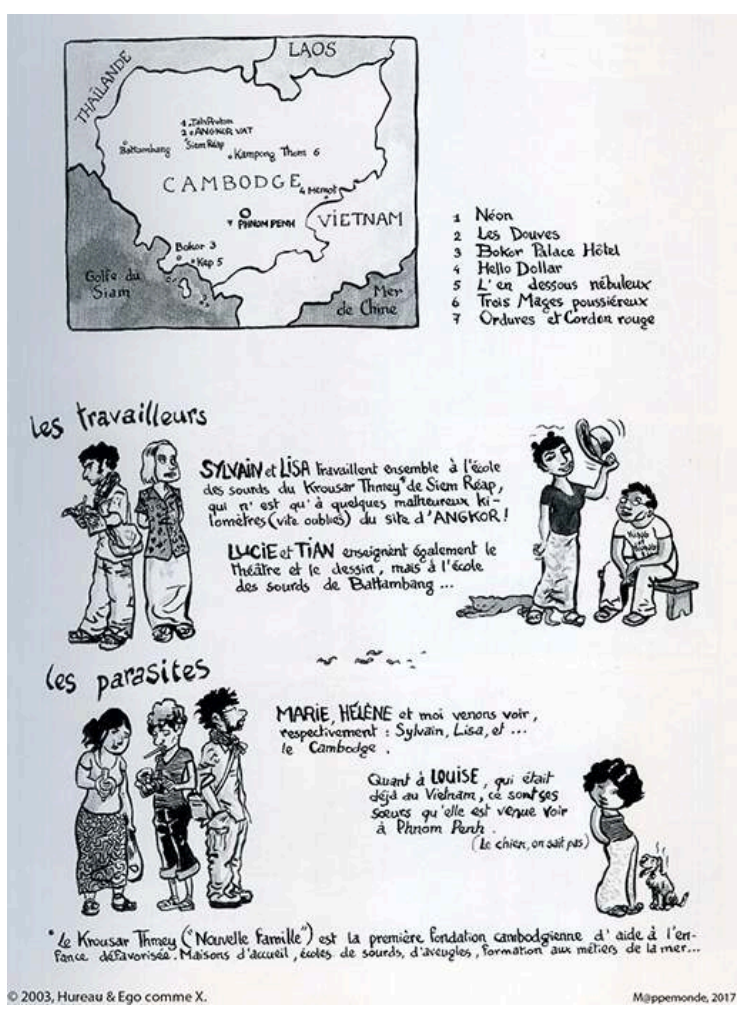

2003, Angoulême, S. Hureau \& Ego comme X, p. 7

La fonction est la même, avec un choix tactique différent, de validation a posteriori de l'ancrage et/ou de la véracité du récit, lorsque la carte est positionnée après celui-ci. C'est le cas pour Brouillard au Pont de Tolbiac de Jacques Tardi interprétant Léo Malet, qui situe l'action dans le tissu urbain du XIII ${ }^{\mathrm{e}}$ arrondissement de Paris ou pour Maus (tome 2) de Art Spiegelman, qui représente le complexe Auschwitz/Birkenau en quatrième de couverture, avec en incrustation un extrait de la carte de l'État de New York, où l'auteur recueille le récit de son père, survivant du camp d'extermination.

Avec le développement de la «BD du réel » (Veschambre, 2011) depuis les années 1990, BD de voyage, de reportage, documentaire, autobiographique, historique, politique..., l'ajout de dossiers documentaires tend à devenir courant. L'auteur y fournit les éléments de contexte utiles, dont les cartes peuvent faire partie, sans alourdir pour autant le récit séquentiel avec des informations qui nuiraient à sa fluidité. A contrario, même si l'on peut y revenir facilement pour se repérer au fil du récit, ce n'est pas la manière la plus dynamique d'accompagner la lecture, ces cartes restant en dehors de l'enchaînement séquentiel des images.

\section{La carte-case au fil du récit}

Comme je l'ai noté précédemment, la carte est généralement délimitée par un cadre, ce qui permet d'en faire une case insérée dans le récit : c'est pour cela que j'ai dénommé carte-case ce deuxième type de carte. Ces cartes-cases s'insèrent dans le récit séquentiel, permettant de suivre le héros, comme dans le Lotus bleu d'Hergés, de montrer la progressive désintégration de l'ex-Yougoslavie (Gorazde de Joe Sacco), d'illustrer la 
variété des espaces, des échelles (Shenzen de Guy Delisle : figure 3) pratiqués par les personnages, mais aussi le caractère (quasi) universel du langage cartographique.

Figure 3. Shenzen
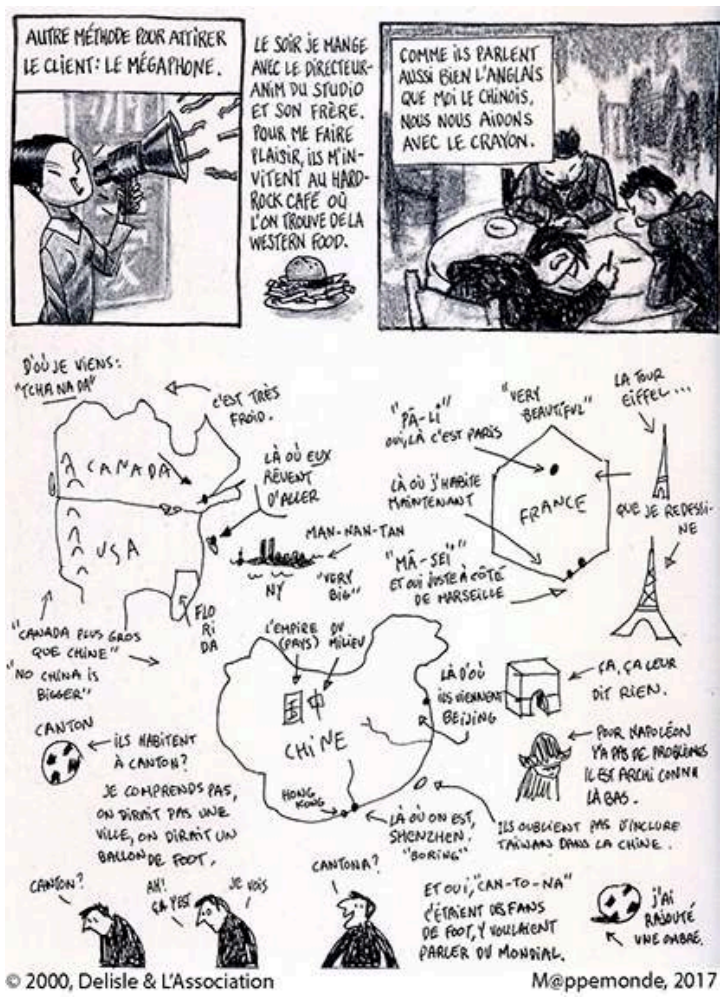

Non paginé.

2000, Paris, G. Delisle \& L'Association

À travers ces quelques exemples, on repère les genres qui apparaissent logiquement les plus étroitement liés à la carte : récits historiques, de science-fiction, reportages ou récits de voyage. À l'inverse, la BD d'humour, où le format strip est fréquent, avec un contexte spatial généralement très simplifié, voire abstrait, est l'un des genres où la carte semble la moins présente (tableau 3).

Tableau 3. BD du corpus 100 selon le genre et la présence ou non de carte

\begin{tabular}{|l|l|l|}
\hline Genre & Nombre BD avec cartes & Nombre BD total \\
\hline Humour & 3 & 10 \\
\hline Aventure & 4 & 12 \\
\hline Fantastique & 6 & 16 \\
\hline Historique & 6 & 15 \\
\hline Science-fiction & 4 & 7 \\
\hline Reportage, récit de voyage & 4 & 6 \\
\hline
\end{tabular}




\begin{tabular}{|l|l|l|}
\hline Policier & 8 & 11 \\
\hline
\end{tabular}

Source : échantillon 100 BD médiathèque du Rize (2014).

Catégorisation en genre : cf. Groensteen T., Ternaux C. (dir.), 1998, La Bédéthèque idéale, Angoulême, Musée de la bande dessinée.

Ces cartes-cases sont présentes de manière inégale dans les deux corpus constitués : de 25 à $60 \%$ des BD avec cartes, selon que l'on se situe dans l'échantillon de 100 BD et dans celui de 50. La suite des recherches pourrait permettre de préciser un ordre de grandeur et de situer la fréquence de ce type de représentation cartographique par rapport aux deux autres repérées.

Dans la suite du texte, j'aurai l'occasion de revenir sur les enjeux graphiques qui sont associés à ces cartes-cases, qui sont celles où l'auteur cherche à associer le plus étroitement récit séquentiel et cartographie.

\section{La carte-objet : élément modificateur du récit ou expression d'un pouvoir}

Il faut également envisager la carte comme objet utilisé par les personnages ou présent dans l'environnement qui est le leur. Cet objet est relativement fréquent dans la bande dessinée, dans plus de la moitié des ouvrages avec cartes de mes deux corpus (tableau 2).

La carte peut être centrale, comme dans La Frontière invisible signée François Schuiten et Benoît Peeters, qui met en scène un personnage de cartographe. Elle peut être plus anecdotique ou poétique, comme dans le Petit Prince interprété par Joann Sfar: Saint-Exupéry y déploie sur le sol une grande carte du ciel bleu nuit, sur laquelle le Petit Prince ne parvient pas à repérer son astéroïde.

30 Manipulée par les personnages, la carte joue toujours un rôle dans le déroulement du récit. Dans le premier tome de la série Bone de JefF Smith, le personnage principal consulte une carte (que l'on ne peut d'ailleurs pas lire), roulée comme un parchemin, juste avant de chuter et de se retrouver dans un univers inconnu (et non cartographié) : la consultation de la carte déclenche le récit.

31 Dans Le Secret de l'Espadon de Edgar P. Jacobs, on peut voir, figuré en pied, le personnage d'olric déplier une carte. Case suivante, au milieu de la bande inférieure de la planche, la main d'olric est en train d'entourer le point stratégique, pendant qu'il déclare: « Mais voyez la carte messieurs ». Carte annonciatrice de l'action de guerre à venir. Une carte qui, à l'échelle de la case et en gros plan, ne peut qu'être extrêmement simplifiée : on ne lit qu'une simple cible dans le détroit d'Ormuz.

Dans une situation analogue, où la carte sert à lire des enjeux géopolitiques, Milton Caniff (Terry et les pirates : dangereuses passions) fait un autre choix. On voit tout d'abord un personnage de capitaine brandir une carte et déclarer au héros «jetez un œil sur cette carte et vous comprendrez très vite ». Au lieu de faire un zoom sur les mains du personnage comme Jacobs, il choisit de passer à la représentation de type carte-case, ce qui permet un luxe de précision, avec tous les attributs de la carte : orientation, échelle, figurés symboliques pour localiser les mines, les propriétés, les voies de transport, les obstacles topographiques... tout ce qui attend le héros sur le terrain.

3 Pour mettre en scène une carte-objet, en faisant bénéficier le lecteur de l'information qu'elle représente, de manière visible et crédible, le choix est a priori limité : soit 
zoomer sur une partie de la carte, avec une information forcément réduite et un cadrage acrobatique, soit changer de représentation pour passer à la carte-case, au risque de l'artifice et de la rupture dans la narration. Je reviendrai sur cette question de la rupture potentielle que représente la carte-case par rapport à la case ordinaire du récit séquentiel.

Outre la carte manipulée par un personnage, un autre grand type de carte-objet peut apparaître ( $1 / 3$ des occurrences de cartes-objets) : la carte murale, plus ou moins lisible en arrière-plan de la scène, souvent partiellement occultée par le cadrage ou les bulles. Chez Hergé, Milton Caniff ou François Schuiten, les cartes murales sont afFichées dans des lieux d'autorité ou de pouvoir (bureaux de policiers, de militaires, de dirigeants...) et représentent des attributs de ce pouvoir.

Cette distinction entre cartes manipulées et cartes affichées, recouvre celle établie par Christian Jacob (1992) entre " carte mobile » et " carte immobile » : ces deux types de cartes jouent un rôle bien différent dans la narration, avec une plus forte présence de la carte mobile (deux tiers des occurrences), qui peut être impliquée directement dans la structure narrative.

Si la carte apparait bien présente dans la bande dessinée, on remarque cependant que son insertion dans le récit séquentiel ne va pas de soi : il apparait difficile de montrer une carte-objet et il semble plus simple de placer une carte de contextualisation en dehors du récit. Cette question de la compatibilité entre l'icône cartographique et le langage de la bande dessinée mérite d'être développée.

\section{La carte dans la BD : entre évacuation et « contamination »}

Depuis un premier article en 1986, Thierry Groensteen est revenu à plusieurs reprises sur l'idée d'une tension introduite dans le récit séquentiel par la présence d'une icône. Il a développé cette théorie à partir de l'exemple du tableau, icône fixe, isolée, alors que les cases sont solidaires et animées par leur succession: "l'image fixe ou tableau apparaît comme une zone de résistance qu'il convient de réduire ou de contaminer par n'importe quel moyen" (Groensteen, 2003, p. 152). Cette théorie me parait s'appliquer a fortiori à la carte, icône qui tranche d'autant plus avec les autres icônes présentes dans une $\mathrm{BD}$ qu'elle ne simule généralement pas la $3^{\mathrm{e}}$ dimension. C'est ainsi que deux tendances sont fréquemment observables à propos de la carte en bande dessinée : son éviction du récit ou sa contamination par le langage de la BD.

\section{Une carte fréquemment évacuée dans le paratexte}

38 On peut donc faire le constat que la carte est fréquemment mise en marge du récit séquentiel. C'est ce qui se produit avec la carte hors-récit, placée en préambule ou en postface, dans ce que l'on peut qualifier de paratexte.

Cette exclusion de la carte va parfois plus loin. Dans le cas de la série de science-fiction, Le Cycle de Cyann de François Bourgeon et Claude Lacroix, les cartes sont reléguées dans un atlas (La Clef des confins), qui présente par ailleurs la faune, la flore, l'architecture, les types d'habillement caractéristiques de la planète. 

l'on en a besoin. Avec cet inconvénient d'introduire une rupture dans le mode de représentation. Dans l'édition couleur du Lotus bleu d'Hergé, les deux cartes-cases qui permettaient de suivre, dans l'édition originale, le parcours asiatique de Tintin ont finalement disparu : elles ont peut-être été jugées trop didactiques ou en rupture avec la narration. Dans le même ordre d'idée, la carte liminaire des Aventures d'Astérix était insérée dans le récit dans l'édition originale d'Astérix le Gaulois (1961), avant de glisser dans le paratexte dès le second album.

Dans mon corpus de $50 \mathrm{BD}$ avec cartes, j'ai regardé de plus près comment ces cartescases s'articulent avec les autres cases. Dans un cas sur deux, la carte-case en question est décalée, voire isolée des autres cases, par différents types de procédés : placée en tête de chapitre (comme dans le tome 1 de The Unwritten, de Mike Carey et Peter Gross), occupant une pleine page ou une double page (comme dans Le Jeu des hirondelles de Zeina Abirached: figure 4), subissant une légère rotation par rapport aux cases environnantes (comme dans Maus 1 de Art Spiegelmann), apparaissant en décalage chromatique (comme dans Dora [tome 1] de Ignacio R. Minaverry) ou, tout simplement, présentant un cadre d'une autre épaisseur ou couleur ou une absence de cadre (comme dans Shenzen de Guy Delisle). 


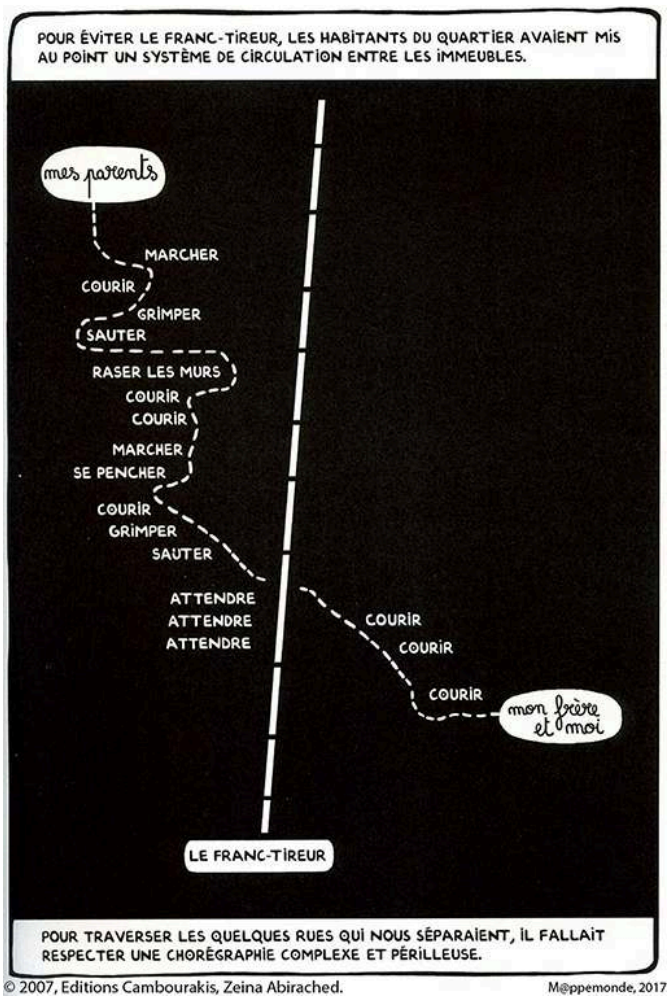

2007, Paris, Éditions Kambourakis, Zeina Abirached, p. 15

Dans ce corpus de 50 BD, $60 \%$ des cartes-représentations (toutes les cartes hors-récit et la moitié des cartes-cases) sont ainsi décalées du récit séquentiel, de manière qu'il n'y ait pas d'ambiguïté quant à la spécificité de ce type d'icône par rapport aux autres.

\section{La carte « contaminée » par le langage BD : une icône hybridée}

L'autre tendance observable est inversement à l'harmonisation entre représentation cartographique et graphisme de la BD. Le premier moyen pour évaluer le degré de " contamination» de la carte est de déterminer si elle a été (re)dessinée ou non par l'auteur.

Dans mon corpus de 50 BD, $70 \%$ des cartes (cartes-représentations ou cartes-objets) sont dessinées et présentent donc un graphisme homogénéisé par rapport aux cases environnantes (tableau 4).

Tableau 4. Typologie des cartes dans le corpus de 50 BD

\begin{tabular}{|l|l|l|l|l|}
\hline & Cartes hors-récit & Cartes-cases & Cartes objets & Cartes \\
\hline Nombre & 32 & 133 & 75 & 239 \\
\hline$\%$ & $14 \%$ & $55 \%$ & $31 \%$ & $100 \%$ \\
\hline Dont cartes (re)dessinées & 20 & 101 & 50 & 171 \\
\hline
\end{tabular}




\begin{tabular}{|l|l|l|l|l|}
\hline \% de cartes (re)dessinées & $63 \% *$ & $76 \%$ & $67 \%$ & $72 \%$ \\
\hline
\end{tabular}

*Plus éloignées de la trame séquentielle proprement dite, les cartes hors récit ne sont dessinées par l'auteur que dans moins de deux cas sur trois, comme si l'on pouvait plus facilement s'éviter en cette position d'adapter l'icône cartographique au graphisme de la BD.

Source : échantillon personnel de 50 BD avec cartes

Les cartes insérées dans Gorazde de Sacco reprennent le langage graphique de l'auteur : on y retrouve les mêmes traits, lettrages, grisés, trames que dans les autres cases et les cartes-cases se fondent ainsi par mimétisme dans la trame séquentielle.

Dans un entretien qu'il m'a accordé à propos de Rural (Veschambre, 2011), Étienne Davodeau m'a parlé de son goût pour les cartes, mais aussi de sa tendance à les redessiner pour homogénéiser les cases. S'il conçoit d'introduire des cartes "brutes ${ }^{6}$, c'est dans un contexte documentaire (comme il peut insérer un extrait de journal, « qui fait preuve »), mais en aucun cas dans une œuvre de fiction.

En dessinant la carte, l'auteur de BD tend à s'afFranchir des codes de la carte «scientifique », qui présente une orientation, une échelle, une légende, un titre... Pour les cartes de Joe Sacco, dont la fonction est clairement informative, la présence d'une échelle ou d'une légende est loin d'être systématique. La marge de manœuvre est encore plus importante quand il s'agit de cartographier des mondes imaginaires. Éditée par l'IGN, pour faire plus «vrai », la carte des Cités obscures ${ }^{7}$ dessinée par François Schuiten ne présente pas de légende : il faut dire que le dessin est imitatif et que les éléments représentés renvoient directement au signifié, sans passer par l'abstraction. Et l'échelle indiquée sur la couverture est en unité « cités obscures».

1 D'autres codes de la cartographie peuvent être ainsi détournés : sur la carte de Louxor dessinée par Jean-Christophe Menu (figure 5), dans l'Association en Égypte, l'échelle graphique est curviligne. 
Figure 5. « Hatshepsout blues » in l'Association en Égypte

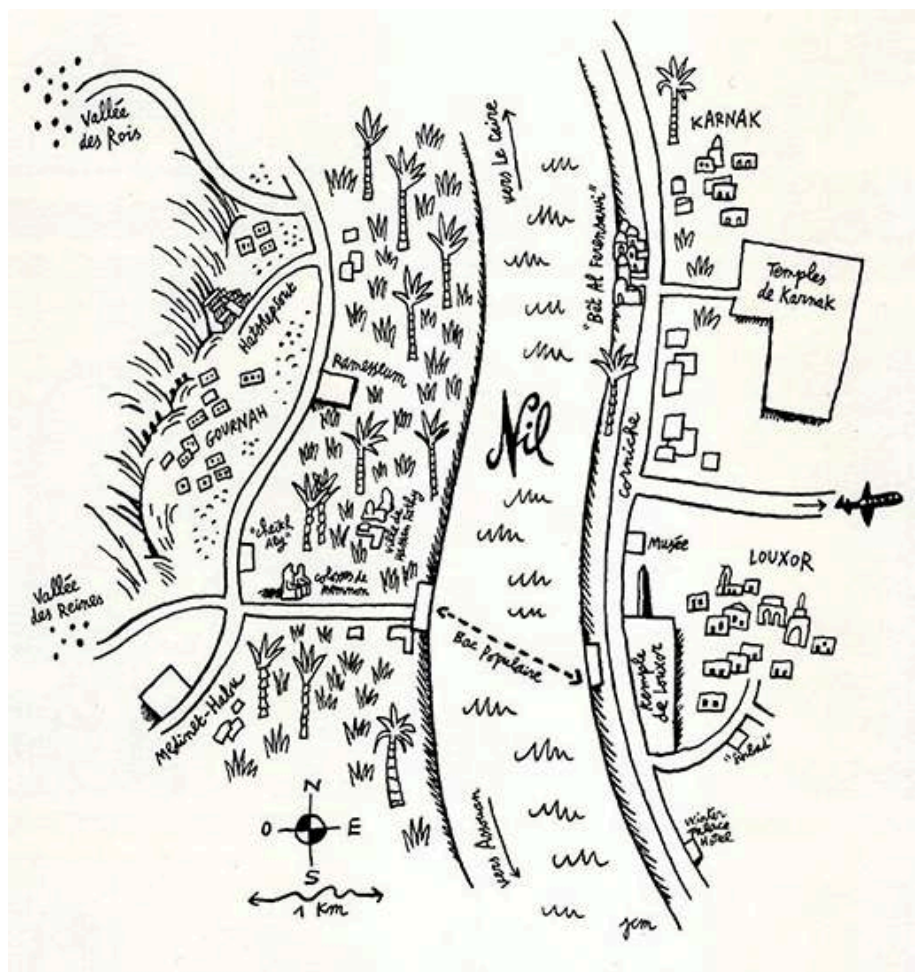

- 1998, Menu \& L'Association

M९ppemonde, 2017

Non paginé.

1998, Paris, J.C. Menu \& L'Association

52 Les formes de « contamination » de la carte par le graphisme BD sont infinies. Cela peut s'opérer également par la surimposition de cases en incrustation, comme sur le parchemin, typique des mondes de la fantasy, dessiné par Goran Skrobonja et Drazen Kovacevic dans La Roue. Ou plus simplement encore, par la surimposition de phylactères ou d'encadrés identiques à ceux que l'on peut lire dans les autres cases, comme dans Vertiges de Quito de Didier Tronchet (figure 6). 
Figure 6. Vertiges de Quito

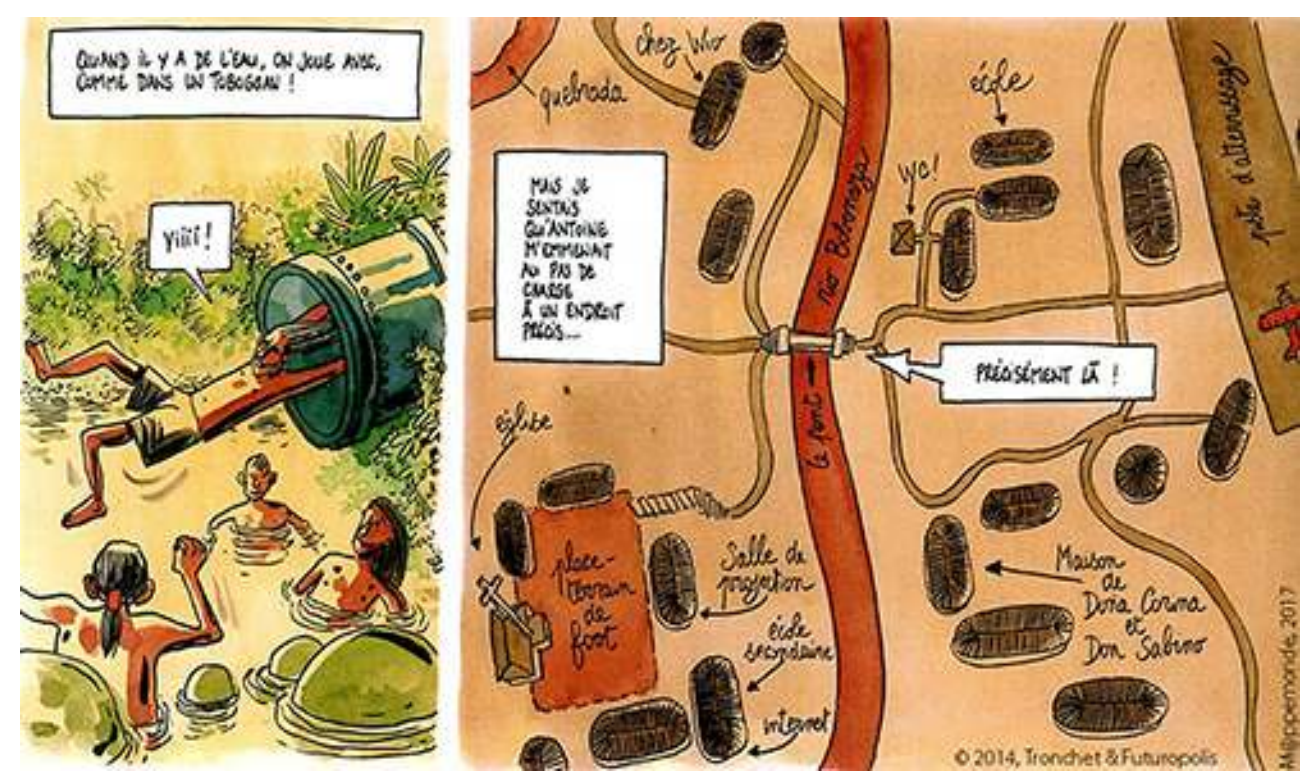

2014, Paris, D. Tronchet \& Futuropolis, p. 62

Les exemples les plus spectaculaires sont ceux où le dessinateur surimpose des éléments simulant la $3^{\mathrm{e}}$ dimension (personnages, décor...) sur un fond de carte à deux dimensions. Le meilleur exemple en est sans doute la carte liminaire des Aventures d'Astérix, où l'on voit une enseigne de la légion romaine plantée en plein cœur de la Gaule, ainsi qu'une loupe permettant le changement d'échelle au niveau du «village peuplé d'irréductibles Gaulois » et des camps romains qui le circonscrivent.

La dernière planche du Piéton du Caire de Golo est, quant à elle, constituée d'un plan du centre-ville, littéralement arpenté par un marchand de lait et un crieur de journaux.

Parmi les nombreuses cartes figurant dans Rural de Étienne Davodeau, celle qui permet de localiser les tracés autoroutiers envisagés est une carte-case hybride : l'opposant interviewé apparaît sur fond cartographique, ce qui rend son commentaire des tracés immédiatement compréhensible, d'autant plus que l'agencement des phylactères souligne et localise les trois options (figure 7).

Figure 7. Rural

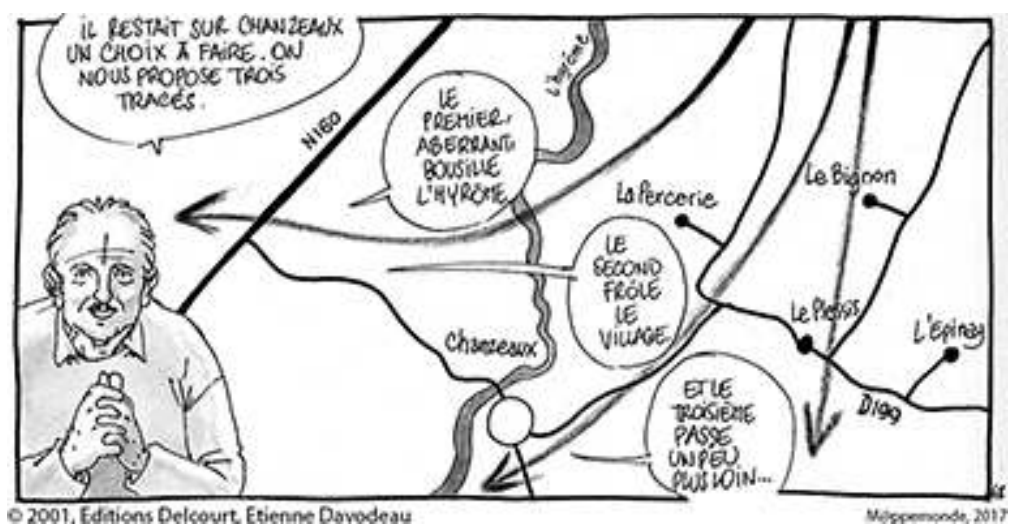

2001, Paris, Éditions Delcourt, Étienne Davodeau, p 74 
Dans la dernière case du Voyage de Caboto dessiné par Lorenzo Mattotti, émergent du portulan aux couleurs chaudes des visages de femmes, de conquistadors et d'Indiens, ainsi que des caravelles. Dans la case précédente, où le portulan sort de l'ombre, on peut lire la dernière phrase du livre : « sur tout visage se dessine la carte d'une vie ».

À propos de cette question de la place des icônes dans la bande dessinée, Thierry Groensteen (1986) écrit : « lorsqu'un tableau est cité dans une BD, les auteurs n'ont de cesse que de l'engager dans un processus d'animation, soit que les figures portraiturées sortent du cadre, soit que le héros de l'aventure y pénètre. Face aux images en soi de la peinture, les dessinateurs de BD se comportent comme s'il s'agissait réellement de fenêtres ouvertes sur le monde ». Voilà qui s'applique tout particulièrement à la carte, telle qu'elle est utilisée dans la bande dessinée, avec les porosités que l'on peut constater : une carte qui fonctionne comme médium privilégié entre le récit séquentiel et le monde, réaliste ou imaginaire, dans lequel il se déploie.

\section{Conclusion}

La BD a généralement besoin d'être ancrée dans un espace pour produire un récit : de ce point de vue, la carte apparaît bien présente, permettant non seulement de crédibiliser ce récit (notamment pour les genres de la fantasy ou de la science-fiction), mais encore de le nourrir. À travers l'analyse de ces deux corpus, j'ai pu établir à la fois la grande proximité entre la carte et la $\mathrm{BD}$, en même temps que les tensions produites par leur rapprochement : il s'agit en efFet d'insérer une icône (carte-objet) dans une icône plus large ou dans une série d'icônes de nature difFérente (une carte-case au milieu d'autres cases), ce qui pose problème et conduit parfois à l'évacuer (carte hors-récit). La plupart du temps, la carte, même gage de réalisme, est dessinée par l'auteur, avec ses propres codes, qui ne renvoient pas à ceux de la carte scientifique, afin de mieux l'intégrer graphiquement. Et quand elle est empruntée à des cartographes patentés, c'est généralement pour la surcharger de signes ou de représentations en trois dimensions. Cette hybridation de la carte par l'auteur de bande dessinée en fait un monde en soi, qui peut alors être investi et habité.

\section{BIBLIOGRAPHIE}

BELLEFROID T. (2008). Le Voyageur de Troy. Entretiens avec Arleston. Toulon : Soleil, 142 p.

GARRIC H. (2007). Portraits de villes. Marches et cartes : la représentation urbaine dans les discours contemporains. Paris : Honoré Champion, 576 p. ISBN 2745315072

GAUMER P. (1996). Les Années Pilote : 1959-1989. Paris: Dargaud, 303 p.

GROENSTEEN T., SMOLDEREN T. (1986). « Tableaux vivants ». Les Cahiers de la BD. n 68, p. 88-97.

GROENSTEEN T. (1999). Système de la bande dessinée. Paris : PUF, 206 p.

Mappemonde, 121 | 2017 
GROENSTEEN T. (2003). Lignes de vie : le visage dessiné. Saint-Égrève : Mosquito, 192 p. ISBN 2908551527

JАСОВ C. (1992). L'Empire des cartes. Approche théorique de la cartographie à travers l'histoire. Paris :

Albin Michel, 537 p.

MCCLoud S. (2007). L'Art invisible. Paris : Delcourt, 232 p. ISBN 9782756009698

MARLET O. (2007). « Voyage dans la bande dessinée à travers quelques cartes ». M@ppemonde, consulté le 16 octobre 2011. https://mappemonde-archive.mgm.fr/actualites/voyage_bd.html VESCHAMBRE V. (2011). «Quand la bande dessinée parle de paysage et de géopolitique locale. Rural! d'Étienne Davodeau ». In TRIVISANI-MOREAU I. (dir.), Paysage politique, le regard de l'artiste, Rennes : PUR, p. 197-210.

\section{ANNEXES}

\section{Éléments du corpus cités dans le texte}

\section{Dans l'ordre d'apparition des titres}

\begin{tabular}{|l|l|l|}
\hline Auteurs & Titres & Années \\
\hline Schuiten/Peeters & Cités obscures (série) & $1983-2014$ \\
\hline Hergé & Les Aventures de Tintin et Milou (série) & $1929-1976$ \\
\hline Goscinny/Uderzo & Les Aventures d'Astérix le Gaulois (série) & $1961-1979$ \\
\hline Pratt & La Ballade de la mer salée & 1975 \\
\hline Fred & Philémon (série) & $1972-2013$ \\
\hline Baladi & Encore un eFrrt & 2009 \\
\hline Loisel/Tripp & Magasin général (série) & $2006-2014$ \\
\hline Bourgeon & Les Passagers du vent (tome 3) & 1981 \\
\hline Arleston/Tarquin & Lanfeust de Troy (tome 1) & 1994 \\
\hline Biagi/Manara & Christophe Colomb & 1992 \\
\hline Hureau & Palace & 2003 \\
\hline Tardi & Brouillard au pont de Tolbiac & $1986 / 1991$ \\
\hline Spiegelman & Maus (I et II) & $2001-2004$ \\
\hline Hergé & Le Lotus bleu & \\
\hline Sacco & Gorazde (série) & 1936 \\
\hline & & 1904 \\
\hline
\end{tabular}




\begin{tabular}{|l|l|l|}
\hline Schuiten/Peeters & La Frontière invisible (1 et 2) & $2002 / 2004$ \\
\hline Sfar & Le Petit Prince & 2008 \\
\hline Smith & Bone (série) & $1995-2005$ \\
\hline Jacobs & Le Secret de l'Espadon (2 tomes) & $1950 / 1953$ \\
\hline CanifF & Terry et les pirates & $1935-1938$ \\
\hline Bourgeon & Le Cycle de Cyann (série) & $1993-2014$ \\
\hline Gibrat & Le Sursis (2 tomes) & $1997 / 1999$ \\
\hline Carey/Gross & The Unwritten (tome 1) & 2011 \\
\hline Abirached & Le Jeu des hirondelles & 2007 \\
\hline Minaverry & Dora (tome 1) & 2012 \\
\hline Delisle & Shenzen & 2000 \\
\hline Davodeau & Rural & 2000 \\
\hline Skrobonja/Kovacevic & La Roue (tome 1) & 1993 \\
\hline Tronchet & Vertiges de Quito & 1998 \\
\hline Menu & L'Association en Égypte (Hatshepsout blues) & 2001 \\
\hline Golo & Mattoti/Zentner & 198 de Caboto \\
\hline
\end{tabular}

\section{NOTES}

1. Ville et BD, ENS de Lyon, 18 sept. 2013.

2. Plusieurs indices tendent à montrer que les bandes dessinées asiatiques présentent des spécificités, par rapport notamment aux types d'enchaînements entre cases (Mac Cloud, 2007).

3. J'ai analysé de manière aléatoire un échantillon de $100 \mathrm{BD}$ issues du fonds de la médiathèque du Rize (à Villeurbanne), fonds constitué depuis 2008.

4. Interview de Jean-Louis Tripp dans DBD, n 9, janv. 2007, p. 54-55.

5. Dans sa version d'origine.

6. On retrouve le même principe dans le volume 5 des Carnets d'Orient de Jacques Ferrandez.

7. On pourra en avoir un aperçu sur http://www.franceculture.fr/emissions/villes-mondes/villemondes-imaginaires-cites-obscures-escale-1-0 


\section{RÉSUMÉS}

Définie comme « art séquentiel », la bande dessinée est un genre dans lequel narration et mise en espace entretiennent des liens privilégiés. D'où la présence fréquente de cartes qui accompagnent le récit $\mathrm{BD}$. Cet article fait le point sur l'affinité entre récit séquentiel et production cartographique, à travers l'analyse de deux corpus de bandes dessinées occidentales. À la fois proche du registre graphique qui est celui de la BD et en rupture avec celui-ci en tant que représentation à deux dimensions, la carte apparaît tantôt évacuée dans le paratexte, tantôt hybridée par le langage séquentiel.

Defined as "sequential art", comics are a genre in which narration and spatiality are closely linked. This explains the frequency of maps accompanying narratives. This article examines the a FFInity between the sequential narrative and the cartographic production through the analysis of two corpuses of Western comics. Both similar graphically to the comic book and clearly difF erentiated as a two dimensional representation, the map is either evacuated in the paratext or hybridized by sequential language.

Considerado como un arte secuencial, el cómic es un género en el que se fusionan narración y espacio, y donde muchas veces están presentes los mapas. Analizando dos conjuntos de comics, este artículo estudia las afinidades entre el relato secuencial de las tiras gráficas y la elaboración de cartografía temática. Los mapas en los comics se presentan de forma contrapuesta apareciendo fuera de la imagen como paratexto en un cartucho, o hibridándose en el lenguaje secuencial de las viñetas.

\section{INDEX}

Thèmes : La mise en carte des récits

Palabras claves : cómic, mapa, viñeta, cuadro, icono

Keywords : comic books, map, panel, outline, icon

Mots-clés : bande dessinée, cadre, carte, case, icône

\section{AUTEUR}

VINCENT VESCHAMBRE

EVS-LAURE, ENSA de Lyon 